\title{
Rabies virus phosphoprotein P5 binding to BECN1 regulates self-replication by BECN1- mediated autophagy signaling pathway
}

\author{
Juan Liu', Min Liao ${ }^{1 *}$, Yan Yan ${ }^{1}$, Hui Yang ${ }^{1}$, Hailong Wang ${ }^{1}$ and Jiyong Zhou ${ }^{1,2}$
}

\begin{abstract}
Background: Rabies virus (RABV) is reported to encode five phosphoproteins (P), which are involved in viral genomic replication, axonal transport, oxidative stress, interferon antagonism, and autophagy induction. However, the functions of the different P proteins are poorly understood.

Methods: Immunofluorescence staining and western blot were performed to detect the autophagy activity, the form of ring-like structure, and the colocalization of BECN1 and P. Co-immunoprecipitation was performed to detect the interaction between $P$ and BECN1. QRT-PCR and TCID 50 assay were performed to detect the replication level of RABV. Small interfering RNA was used to detect the autophagy signaling pathway.

Results: We found that P5 attaches to N-terminal residues 1-139 of BECN1 (beclin1) on the BECN1 ring-like structure through amino acid residues 173-222 of P5. Subsequently, we found that P5-induced autophagosomes did not fuse with lysosomes. Becn1 silencing did not recover P5 overexpression-induced promotion of RABV replication. Mechanistically, RABV protein PAN82 (P5) induced incomplete autophagy via the BECN1-mediated signaling pathway.

Conclusions: Our data indicate that P5 binding to the BECN1 ring benefits RABV replication by inducing BECN1 signaling pathway-dependent incomplete autophagy, which provides a potential target for antiviral drugs against RABV.
\end{abstract}

Keywords: Rabies virus phosphoprotein P5, Beclin1, Binding domain, Incomplete autophagy, Viral replication

\section{Background}

Rabies is associated with severe neurological symptoms and a high mortality rate, causing over 59,000 human deaths worldwide each year [1]. Rabies virus (RABV), belonging to the Rhabdoviridae family, is a single nonsegmented negative-stranded RNA virus with genome of $12 \mathrm{~kb}$. The RABV genome encodes a nucleoprotein $(\mathrm{N})$, phosphoprotein $(\mathrm{P})$, matrix protein $(\mathrm{M})$, glycoprotein (G), and RNA polymerase (L) [2]. The RABV M protein

\footnotetext{
* Correspondence: liaomin4545@zju.edu.cn

${ }^{1} \mathrm{MOA}$ Key Laboratory of Animal Virology, Center for Veterinary Sciences, Zhejiang University, 866 Yuhangtang Road, Hangzhou, Zhejiang 310058, PR China

Full list of author information is available at the end of the article
}

induces apoptosis by targeting mitochondria [3]. The viral protein $\mathrm{P}$ is a multifunctional protein that is involved in viral transcription and replication [4]. The $\mathrm{P}$ protein-dynein LC8 interaction is involved in the axonal transport of rabies virus along microtubules through neuronal cells [5]. The interaction of RABV P protein with complex I in mitochondria causes mitochondrial dysfunction, increased generation of reactive oxygen species (ROS), and oxidative stress [6]. In addition, the interaction of RABV P protein with the focal adhesion kinase and nucleolin positively regulates viral replication [7]. However, RABV P protein also directly binds to the interferon-induced promyelocytic leukemia protein, 
which inhibits viral replication [8]. P protein's interaction with both cell division cycle 37 (CDC37) and heat shock protein 90 (HSP90) promoted self-stability [9]. RABV P binding to beclin1 (BECN1) can induce incomplete autophagy through the caspase2 (CASP2)-mediated signaling pathways to promote viral genome replication [10]. RABV P also interacts with signal transducer and activator of transcription 1 (STAT1) to counteract interferon (IFN) signaling by creating both cytoplasmic and nuclear blocks for STAT1 [11]. RABV $P$ protein, via an interferon antagonist interaction with activated STAT3, inhibits membrane glycoprotein 130 (GP130) receptor signaling to generate optimal cellular conditions for viral replication and spread [12].

The RABV P protein is phosphorylated by a RABV protein kinase and protein kinase $C$, forming different phosphorylated versions of the $P$ protein [13]. The full length $P$ and small $\mathrm{P}$ proteins $\mathrm{P} 2, \mathrm{P} 3, \mathrm{P} 4$, and $\mathrm{P} 5$, are translated from downstream in-frame AUG initiation codons by a leaky scanning mechanism [14]. These small $P$ proteins have different subcellular localizations. The small $\mathrm{P}$ proteins P3, P4, and P5 are located in the nucleus because of the nuclear localization signal (NLS) located in the Cterminal part of the protein (amino acids 172-297), whereas the cytoplasmic distributions of $\mathrm{P}$ and $\mathrm{P} 2$ result from a CRM1 nuclear export signal (NES) in the Nterminal part of the protein [15]. Thus, the functions of these small P proteins have not been fully determined.

In the present study, we aimed to determine the function of the small $\mathrm{P}$ protein $\mathrm{P} \Delta \mathrm{N} 82$ (P5). The results showed that $\mathrm{P} 5$ binding to the $\mathrm{BECN} 1$ ring-like structure induced incomplete autophagy via the BECN1 mediated signaling pathway, which promotes RABV replication. Our study highlights the role of viral protein P5 in modulating RABV replication.

\section{Materials and methods Cells and viruses}

Mouse neuroblastoma N2a cells (CCL-131) and human embryonic kidney $293 \mathrm{~T}$ cells (CRL-3216) from ATCC were maintained in Dulbecco's modified Eagle's medium (DMEM; Gibco, Carlsbad, CA, USA) supplemented with $10 \%$ heat-activated fetal bovine serum (Gibco/Invitrogen, Carlsbad, CA, USA), $100 \mathrm{U}$ of penicillin $\mathrm{mg} / \mathrm{ml}$, and 100 $\mathrm{mg}$ of streptomycin/ml. RABV strains HEP-Flury and CVS-11 were stored in our laboratory and were propagated in N2a cells. Briefly, N2a cells were infected with RABV at a multiplicity of infection (MOI) of 2. The infected cells were grown in fresh medium at $37^{\circ} \mathrm{C}$ and $5 \% \mathrm{CO}_{2}$ for the indicated times.

\section{Antibodies and reagents}

Rabbit anti-LC3A/B (4108), anti-p-AKT (Ser473) (4060), anti-AKT (4691), and anti-p-MTOR (Ser2448; 5536), anti-MTOR (2983), anti-p-AMPK (4185), anti- AMPK
(2532), anti-p-ERK (4370), anti- ERK (4695), anti-p-P38 (4511), and anti-P38 (9212) antibodies were purchased from Cell Signaling Technology (Beverly, MA, USA). Rabbit anti-P62 (3340-1) antibody was purchased from Epitomics (Burlingame, CA, USA). Rabbit anti-CASP2 (ab179520), anti-ULK1 (ab128859), and anti-ATG5 (ab108327) antibodies were purchased from Abcam (Cambridge, MA, USA). Mouse anti-ATG7 (sc-376,212) and anti-BECN1 (sc-48,341) antibodies were purchased from Santa Cruz Biotechnology (Santa Cruz, CA, USA). Mouse anti-Flag (clone M2) (F1804) mAb, wortmannin (W1628), and 3-MA (M9281) were purchased from Sigma-Aldrich (St. Louis, MO, USA). Rabbit anti-MYC (R1208-1) and anti-GAPDH/glyceraldehyde-3-phosphate dehydrogenase (EM1101) antibodies were purchased from Huaan Biological Technology (Hangzhou, China). Mouse monoclonal antibodies (mAbs) recognizing the $\mathrm{N} / \mathrm{P}$ proteins of $\mathrm{RABV}$ were produced in our laboratory [16]. Fluorescein isothiocyanate (FITC)-labeled goat anti-mouse IgG (62-6511) and Alexa Fluor 546-conjugated anti-rabbit IgG (A10036) were obtained from Thermo Scientific (Waltham, MA, USA). Secondary antibodies comprising horseradish peroxidase-labeled anti-mouse (074-1806) or anti-rabbit (074-1506) IgG were purchased from Kirkegaard \& Perry Laboratories (Millford, MA, USA). Adenovirus expressing mCherry-GFP-LC3B fusion protein (C3011), cell lysis buffer NP-40 (50 mM Tris pH 7.4, $150 \mathrm{mM} \mathrm{NaCl}, 1 \% \mathrm{NP}-40$ ) (P0013F) and phenylmethyl sulfonylfluoride protease inhibitor (ST505) were purchased from Beyotime (Shanghai, China). Earle's balanced salt solution (EBSS) (14155-063) was purchased from Gibco. The jetPRIME cell transfection kit (PT-114-07) was purchased from Polyplus Transfection (Sébastien Brant, Illkirch, France).

\section{Plasmids construction and transfection}

The specific primers used to make the constructs generated in this study are listed in Table S1. The truncated $P$ genes were amplified from the cDNA of HEP-Flury (Accession: AB085828.1) and cloned into pCMV-N-Flag (Clontech, Mountain View, CA, USA, 635688) or pCMV-N-Myc (Clontech, 635,689). The truncated Becn1 genes were amplified from the cDNA of Mus musculus Becn1 (Accession: NM_019584.3) and cloned into pCMV-N-Myc. The other plasmids Flag-P (full-length) and Myc-BECN1 were stored in our laboratory. The siRNA targeting mouse Becn1 gene and shRNA targeting mouse Akt, Mtor, Ampk, Mapk genes were also synthesized by Genepharma (Suzhou, China). The siRNA or shRNA and all the plasmids were transfected into HEK293T or N2a cells using the jetPRIME kit according to the manufacturer's instructions. 


\section{Confocal microscopy}

HEK293T or N2a cells were grown to 70\% confluence on a confocal plate (In Vitro Scientific, Sunnyvale, CA, USA) and then transfected with the indicated plasmids or infected with RABV $(\mathrm{MOI}=2)$ for $24 \mathrm{~h}$. The cells were washed with phosphate-buffered saline (PBS), fixed, and permeabilized with $4 \%$ paraformaldehyde in $\mathrm{PBS}$ at $4{ }^{\circ} \mathrm{C}$ for $20 \mathrm{~min}$, and then incubated with the appropriate primary and secondary antibodies. The nuclei were stained with 4, 6-diamidino-2phenylindole (DAPI). Fluorescence signals were scanned under a Zeiss LSM 780 laser confocal microscopy (Zeiss, Oberkochen, Baden-Württemberg, Germany).

\section{Western blotting}

Cells were harvested and lysed with NP-40 buffer containing phenylmethyl sulfonyl fluoride at $4{ }^{\circ} \mathrm{C}$ for $2 \mathrm{~h}$. Protein samples were separated by $12 \%$ sodium dodecyl sulfate polyacrylamide-gel electrophoresis and were transferred onto nitrocellulose membranes. 5\% nonfat dry milk containing $0.1 \%$ Tween 20 was used to block the nonspecific binding sites for $1 \mathrm{~h}$ at room temperature, and then the membranes were incubated with primary antibody at $4{ }^{\circ} \mathrm{C}$ overnight, followed by the appropriate secondary antibody for $1 \mathrm{~h}$ at room temperature. The blots were developed using a SuperSignal West Femto Substrate Trial Kit (Thermo Scientific, 34,096) according to the manufacturer's protocol. Images were captured using FluorChemm $\mathrm{M}$ chemiluminescent imaging system (Protein Simple, Santa Clara, CA, USA) and ImageJ software (National Institutes of Health, Bethesda, MD, USA) was used to quantify the intensity of the immunoreactive protein bands.

\section{Co-immunoprecipitation}

Transfected HEK293T cells were lysed for $2 \mathrm{~h}$ with NP40 buffer containing $1 \mathrm{mM}$ phenylmethyl sulfonylfluoride protease inhibitor at $4{ }^{\circ} \mathrm{C}$. The insoluble fractions were removed after the cell lysates were centrifuged at $12,000 \mathrm{~g}$ for $10 \mathrm{~min}$ at $4{ }^{\circ} \mathrm{C}$. The soluble fractions were incubated with the indicated antibodies overnight at $4{ }^{\circ} \mathrm{C}$. Fresh protein A/G agarose (Santa Cruz Biotechnology, sc-2003) was then added at $4{ }^{\circ} \mathrm{C}$ for $8 \mathrm{~h}$ before washing with PBS. The eluted proteins were subjected to western blotting analysis.

\section{QRT-PCR}

Quantitative real-time reverse transcription polymerase chain reaction (qRT-PCR) analyses of the mRNA transcripts and anti-genomic RNA were performed as previously described [17]. Briefly, Total RNA was extracted from N2a cells using the TRIZOL Reagent (SuperfecTRI $^{\text {is }}$, 3101-100; Shanghai Pufei Biotech Co., Ltd., Shanghai, China) according to the manufacturer's instructions. The total RNA was reverse transcribed into
cDNAs using a RevertAid RT Reverse Transcription Kit (Thermo Scientific, K1691). The qRT-PCR specific primers used are as follows: upstream primer 5'-AAGGAGTTGAATGACAGGGTGCCA-3' and downstream primer 5'-ACT TGGGATGGTTCGAAAGGAGGA-3' for the RABV anti-genome (115 bp in length), upstream primer 5' -AGCAGCAATGCAGTTCTTTGAGGG-3' and downstream primer $5^{\prime}$-TTGTCAGTTCCATGCC TCCTGT CA-3' for the RABV $N$ gene (164 bp in length), and upstream primer $5^{\prime}$-TCAACAGCAACTCC CACTCTTCCA-3' and downstream primer5'-ACCCTG TTGCTGTAGCCGTATTCA-3' for Gapdh gene (92 bp in length). The qPCR reaction was performed using ChamQ $^{\text {sm }}$ Universal SYBR ${ }^{\circ}$ qPCR Master Mix (Vazyme Biotechnology, Q711-02, Nanjing, China) and a LightCycler96 real-time PCR system (Roche, Basel, Switzerland). The qPCR conditions were as follows: $95^{\circ} \mathrm{C}$ for $300 \mathrm{~s} ; 40$ cycles of $95^{\circ} \mathrm{C}$ for $10 \mathrm{~s}$ and $60^{\circ} \mathrm{C}$ for $30 \mathrm{~s} ; 95^{\circ} \mathrm{C}$ for $10 \mathrm{~s}, 65^{\circ} \mathrm{C}$ for $60 \mathrm{~s}$ and $97^{\circ} \mathrm{C}$ for $1 \mathrm{~s} ; 37^{\circ} \mathrm{C}$ for $30 \mathrm{~s}$. Quantitative analysis was performed using the LightCycler96 software with a relative quantification method $(\Delta \Delta \mathrm{Ct})$ to analyze the levels of viral $N$ mRNA and anti-genomic RNA.

\section{Statistical analysis}

Statistically significant differences between groups were determined using one-way analysis of variance (ANOVA) with the Tukey Multiple Comparison Test and using GraphPad Prism 5 software (GraphPad Software, Inc., La Jolla, CA, USA). A $P$ value of less than 0.05 was considered statistically significant.

\section{Results}

Residues 173-222 of the RABV P protein form an autophagy-inducing domain

Our previous report discovers that the RABV P protein induces incomplete autophagy [10], however, the domain responsible for this incomplete autophagy induction is unknown. To identify the functional domain of the $\mathrm{P}$ protein required for autophagy induction, we constructed six truncated $\mathrm{P}$ protein mutants (Fig. 1a). $293 \mathrm{~T}$ cells were transfected with the plasmids pCMV-N-Flagtagged $\mathrm{P}, \mathrm{P} \Delta \mathrm{C} 75, \mathrm{P} \Delta \mathrm{C} 125, \mathrm{P} \Delta \mathrm{N} 19$ (P2), P $\Delta \mathrm{N} 52$ (P3), $\mathrm{P} \Delta \mathrm{N} 68$ (P4), and $\mathrm{P} \Delta \mathrm{N} 82$ (P5), respectively. Western blotting assay revealed that the level of endogenous LC3-phosphatidylethanolamine conjugate (LC3-II) was dramatically increased in cells transfected with all truncated P mutants except for those transfected with $\mathrm{P} \Delta \mathrm{C} 125$ compared with the empty vector transfected cells, and chloroquine (CQ), a lysosomal proteolysis inhibitor, as a control for autophagic flux (Fig. 1b; $P<0.05$, $P<0.001)$. Moreover, all truncated $\mathrm{P}$ proteins caused no significant increases in autophagy associated proteins autophagy related (ATG)5, ATG7, Unc-51 like autophagy 
a

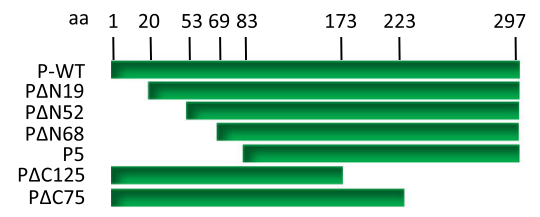

b

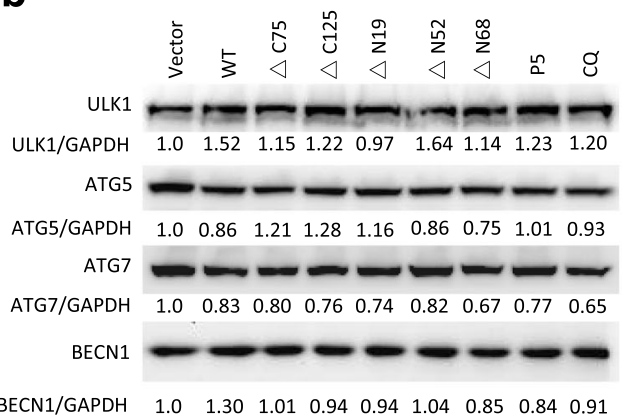

$\begin{array}{llllllllll}\text { BECN1/GAPDH } & 1.0 & 1.30 & 1.01 & 0.94 & 0.94 & 1.04 & 0.85 & 0.84 & 0.91\end{array}$
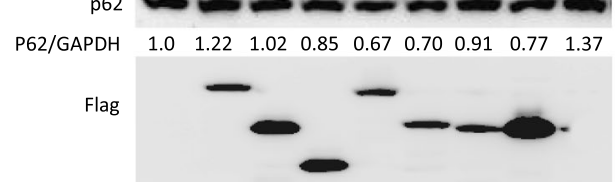

LC3 I

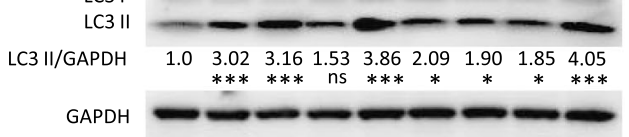

d

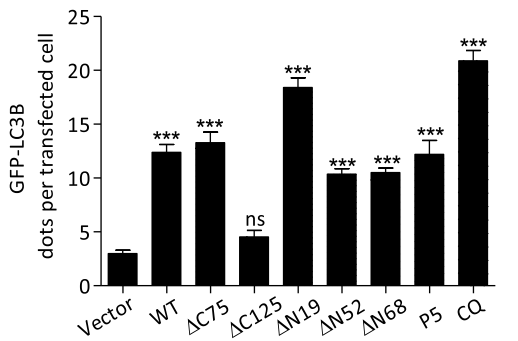

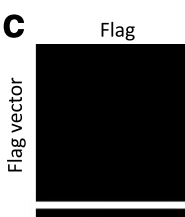
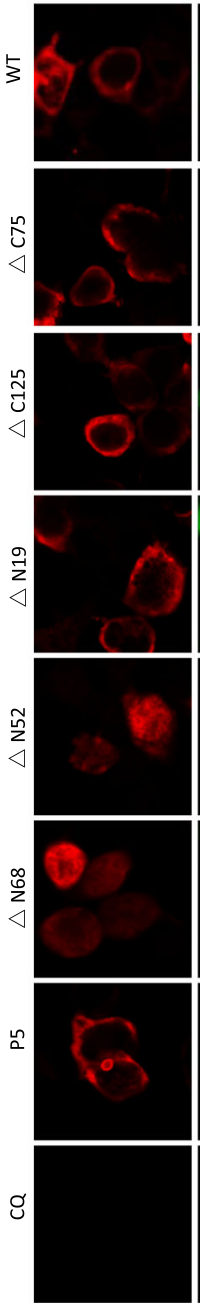

Merge
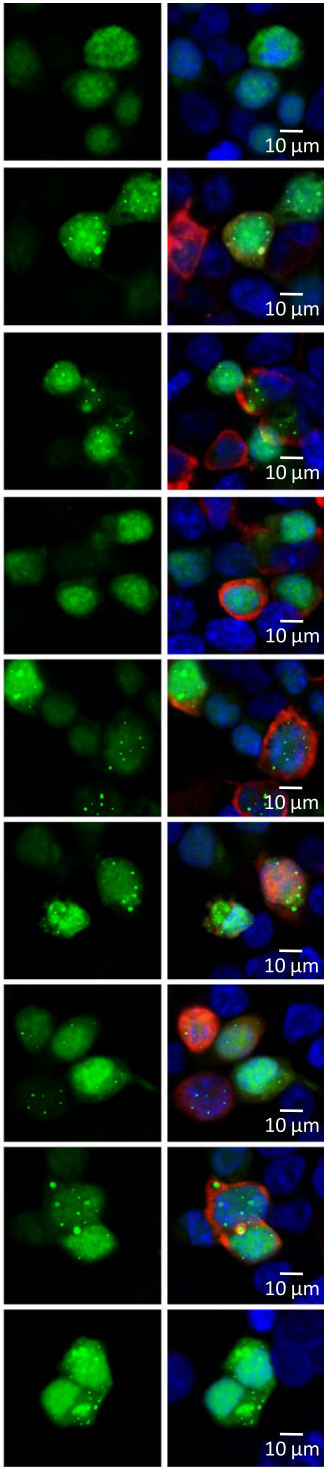

Fig. 1 Truncated P proteins induce autophagy. a Schematic diagram of the full-length and truncated P proteins. b HEK293T Cells were transfected with the plasmids containing the truncated $P$ genes for 48 h, harvested, and analyzed using western blotting using mouse anti-ATG7, anti-Flag mAb, rabbit anti-LC3A/B, anti-ATG5, anti-BECN1, anti-P62, anti-ULK1, and anti-GAPDH antibodies. c HEK293T cells were cotransfected with GFP-LC3B and the plasmids containing the truncated $P$ genes for $24 \mathrm{~h}$, fixed, and immunostained with mouse anti-Flag antibodies (red), and then visualized using confocal microscopy. DAPI (blue) was used to stain nuclear DNA. Scale bar: $10 \mu \mathrm{m}$. $\mathbf{d}$ The graph shows the quantification of autophagosomes by taking the average number of dots in 50 cells. Means and SD (error bars) of three independent experiments are indicated (*, $P<0.05$; $^{* *}, P<0.01$; $\left.^{* *}, P<0.001\right)$

activating kinase 1 (ULK1), BECN1, and autophagic degradation substrate sequestome 1 (also known as P62) levels (Fig. 1b). Consistently, confocal observation also revealed green fluorescent protein (GFP)-LC3B punctas were dramatically increased in cells transfected with all truncated $\mathrm{P}$ mutants except for those transfected with $\mathrm{P} \triangle \mathrm{C} 125$ compared with the empty vector transfected cells (Fig. 1c and d; $P<0.001$ ), however, there were no significant differences in GFP-LC3B punctas caused by all truncated $\mathrm{P}$ proteins after these cells were treated with CQ (Fig. S1). Therefore, these data showed that a P protein containing C-terminal residues aa 173-222 was responsible for autophagic activity.

Phosphoprotein P5 forming ring-like structures induces autophagosomes accumulation

Small phosphoprotein P5 contains residues aa 83-172, 173-222, and 223-297 of full-length $P$ protein. 
Interestingly, a ring circle-like structure was observed in N2a cells transfected only with the P5 mutant but not in N2a cells either transfected individually with other P protein mutants or co-transfected with P5 and other P protein mutants (Fig. 1c, Fig. 2a and Fig. S2). Similarly, the ring circle-like structure was observed in N2a cells co-transfected with both Flag-P5 and Myc-P5 (Fig. 2b), indicating that only P5 could form the ring-like structure. Moreover, the number of GFPLC3B puncta autophagosomes surrounded by the P5 ringlike structure increased significantly in N2a cells cotransfected with GFP-LC3B and Flag-P5 in comparison with N2a cells cotransfected with Flag-vector and GFP-LC3B (Fig. 2c). Collectively, these data demonstrated that the P5 ring-like structure induced autophagosomes accumulation.

Incomplete autophagic vesicles are induced by P5 protein To further investigate the relationship between autophagosomes and P5, N2a cells were cotransfected with FlagP5 and GFP-LC3B, and labeled with LysoTracker Red. As expected, the number of GFP-LC3B puncta autophagosomes increased markedly and did not colocalize with LysoTracker Red in Flag-P5-transfected N2a cells compared with EBSS-treated N2a cells (Fig. 3a), indicating that the autophagosomes did not fuse with acidic compartments after P5 transfection. To rule out the possibility that autophagosomes fused with lysosomes but were not efficiently acidified in the transfected cells, we investigated the colocalization of GFP-LC3B with lysosomal associated membrane protein 1 (LAMP1) in Flag-P5transfected N2a cells. GFP-LC3B puncta did not colocalize with LAMP1 in Flag-P5-transfected N2a (Fig. S3). These data suggested that autophagosomes did not efficiently fuse with lysosomes in Flag-P5-transfected cells.

In addition, we used adenovirus that expressed mCherry-GFP-LC3B, which was used to discriminate autophagosomes (expressing both mCherry and GFP fluorescent) from acidified autolysosomes (expressing red fluorescentonly) to determine the function of P5 in autophagosome maturation. N2a cells were transfected with Flag-P5 plasmids for $12 \mathrm{~h}$, and infected with the adenovirus. In Flag vector transfected cells, few yellow puncta autophagosomes could be detected after adenovirus infection (Fig. 3b). In contrast, in Flag-P5 transfected cells, we observed the accumulation of yellow puncta autophagosomes but a low number of mcherry puncta autophagosomes, suggesting impaired autophagosome fusion with lysosomes. These results implied that P5 protein was responsible for the observed incomplete autophagic induction.

The protein $\mathrm{P} 5$ attaches to the BECN1 ring-like structure by interaction with BECN1

We previously demonstrated that the RABV P protein could interact with BECN1 [10]. To identify whether

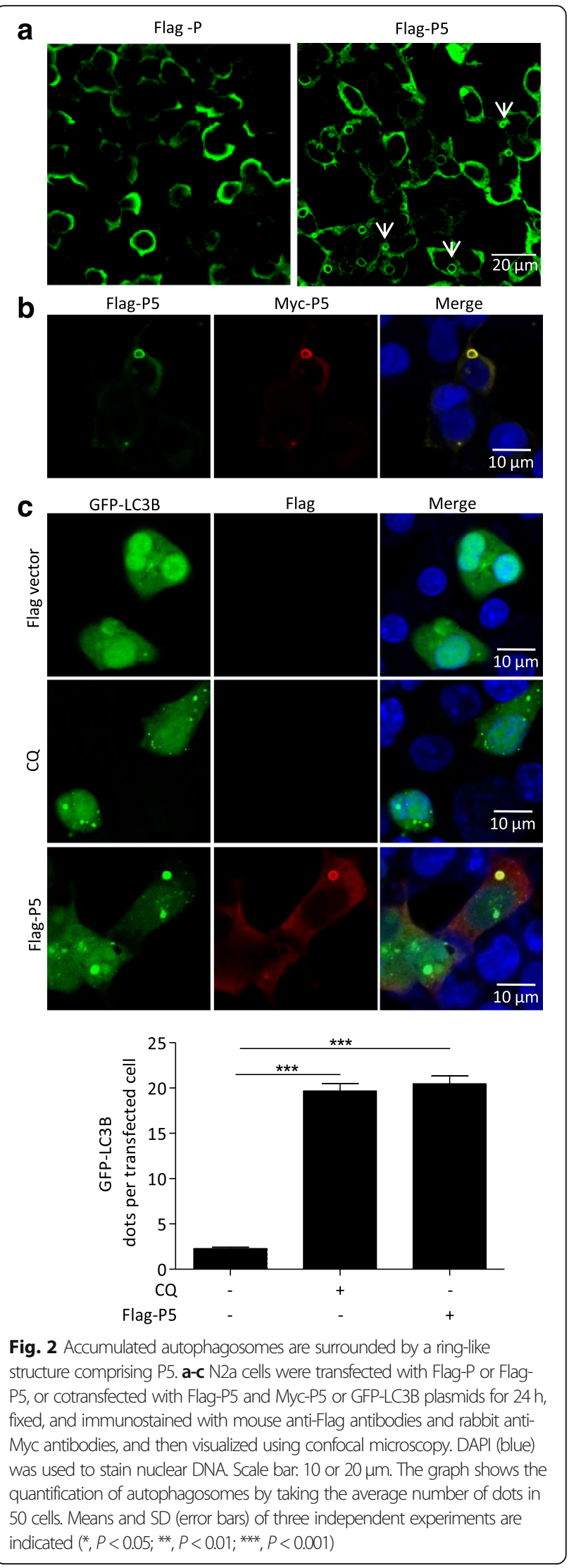




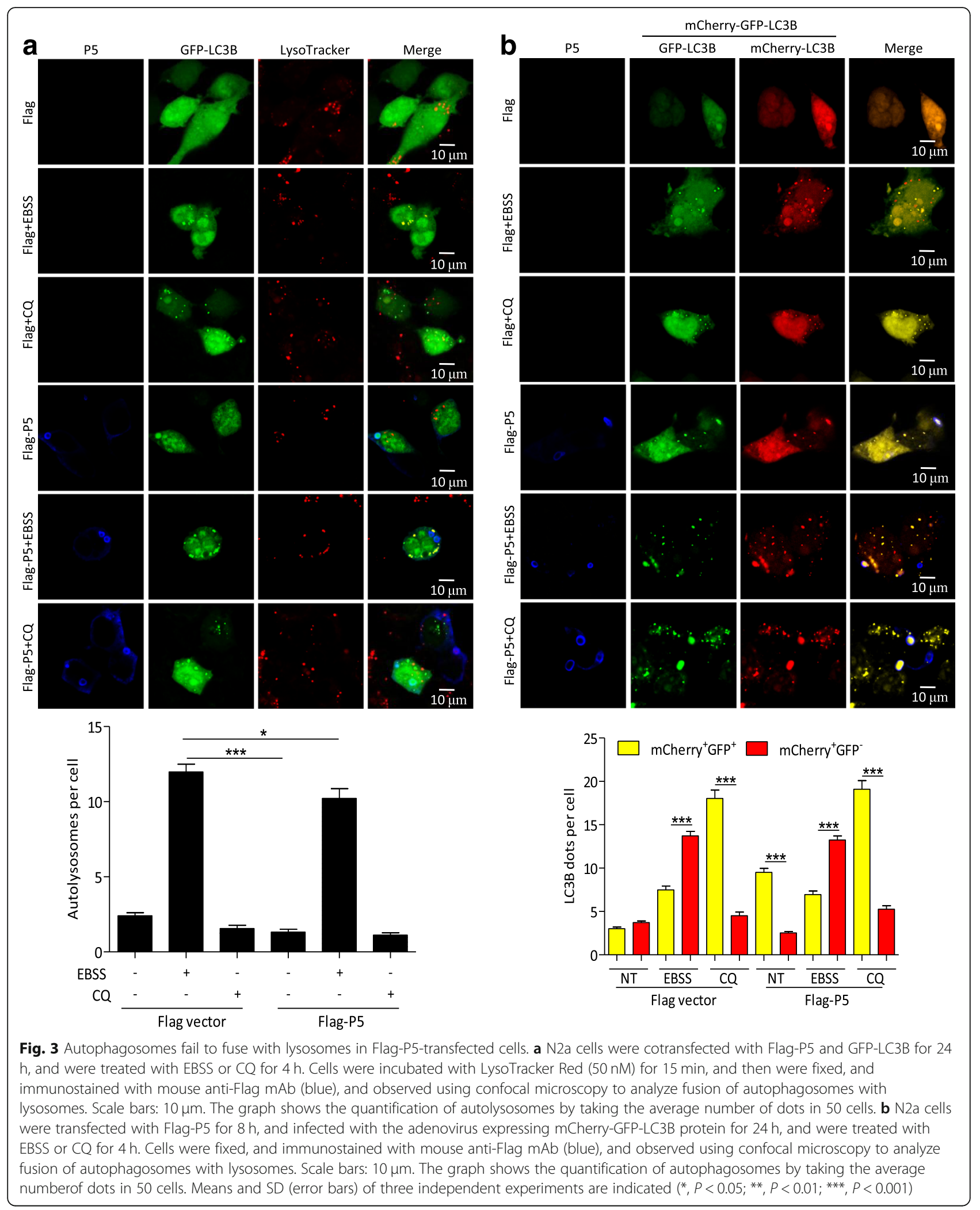


BECN1 binding to the $\mathrm{P}$ protein involves $\mathrm{P}$, N2a cells were cotransfected with Myc-BECN1 and Flag-P5 or Flag- $\mathrm{P} \Delta \mathrm{C} 75$, Flag- $\mathrm{P} \Delta \mathrm{C} 125$, Flag- $\mathrm{P} \Delta \mathrm{N} 19$, Flag- $\mathrm{P} \Delta \mathrm{N} 52$, and Flag-P $\Delta \mathrm{N} 68$, respectively. Confocal microscopy showed that the BECN1 colocalized with the full-length $\mathrm{P}$ and the P mutants except for P $\Delta \mathrm{C} 125 \mathrm{P} 5$ mutant, notably, the $\mathrm{P} 5$ formed ring-like structure had stronger localization with $\mathrm{BECN1}$ ring-like structure compared with $\mathrm{P}$ ring-like structure (Fig. 4a, and Fig. S4). Similarly, endogenous P protein colocalized with $\mathrm{BECN} 1$ to form the ring-like structure in RABV infected cells, and interestingly, the ring-like structure was not observed after Becn1 gene was knocked down, suggesting that BECN1 was necessary for RABV infection to form the ring-like structure (Fig. $4 \mathrm{~b}$ ). Subsequently, a co-immunoprecipitation assay (Co-IP) was performed to further analyze whether the colocalization involves protein-protein interactions. The Co-IP data demonstrated that full-length and all truncated $\mathrm{P}$ proteins except $\mathrm{P} \triangle \mathrm{C} 125$ could immunoprecipitate BECN1, and that P5 showed stronger binding to BECN1 than the other truncated $\mathrm{P}$ mutants (Fig. 4c). In addition, surprisingly, P5's binding ability to BECN1 was stronger than that of the full-length protein (Fig. 4c). To identify the P protein binding domain of BECN1, Myc-tagged truncation mutants of BECN1 (1-351aa, 139-351aa, and 139-448aa) were constructed and transfected into $293 \mathrm{~T}$ cells (Fig. 4d). Confocal microscopy analysis showed that only the 1-351aa BECN1 mutant formed the ring-like structure, and the $\mathrm{P}$ protein colocalized with the ring-like structure and the 1-351aa BECN1 mutant (Fig. 4e). Further co-IP experiments showed that only 1-351aa BECN1, but not 139-351aa BECN1 and 139-448aa BECN1, interacted with $P$ protein (Fig. 4f), revealing that first $139 \mathrm{~N}$-terminal residues of BECN1 are responsible for interacting with P. Collectively, these data confirmed that RABV protein $\mathrm{P}$ attached to the $\mathrm{BECN} 1$ ring-like structure by residues $173-222$ of $\mathrm{P}$ binding to $\mathrm{N}$-terminal residues $1-139$ of BECN1.

\section{P5 binding to BECN1 ring-like structure promoted RABV replication}

To determine the effect on RABV replication of P5 binding to the $\mathrm{BECN1}$ ring-like structure, we investigated the dynamics of RABV infection under condition of P5 overexpression. N2a cells transfected with Flag-P5 for $12 \mathrm{~h}$ were infected with RABV. We found that the level of viral $\mathrm{N}$ protein, viral $N$ mRNA, viral anti-genomic RNA, and infectious RABV progeny were all significantly increased; however, when Becn1 was knocked down using two short interfering RNAs (siBecn1), there was a detectable downregulation of viral $\mathrm{N}$ protein, viral $N$ mRNA, viral anti-genomic RNA, and infectious RABV progeny in the absence or presence of P5 (Fig. 5a-e, $P<0.05$, 0.01 , or 0.001 ), suggesting a positive role of P5 in regulating RABV infection dependent of BECN1. In addition, to further confirm whether the effect of the ring-like structure on RABV replication was dependent of autophagy induction, we also detected the level of viral $\mathrm{N}$ protein in presence of protein P5 together with the autophagy inhibitor 3-methyladenine (3-MA), or wortmannin treatment. The results showed 3-MA or wortmannin treatment significantly inhibited the level of viral $\mathrm{N}$ protein compared with that in non-treated P5 group (Fig. $5 f$ and $\mathrm{g}, P<0.01$ or 0.001$)$. Collectively, these data demonstrated that RABV replication hijacked BECN1 by P5 binding to the BECN1 ring-like structure.

\section{$\mathrm{P} 5$ binding to the $\mathrm{BECN} 1$ ring-like structure regulates RABV replication via the BECN1-mediated signaling pathway}

To further investigate the BECN1-dependent signaling pathway through which P5 regulates RABV replication, we examined whether BECN1, AMP-activated protein kinase (AMPK), CASP2, protein kinase B (AKT), mammalian target of rapamycin (MTOR), and mitogen activated protein kinases MAPKs [extracellular signal-regulated kinase (ERK), P38] levels changed during overexpression of P5. Western blotting analysis showed that P5 dramatically upregulated the phosphorylation (p) level of AKT, MTOR, AMPK, ERK1/2, and P38, and reduced the CASP2 level; however, it did not affect the total amount of these proteins nor BECN1 levels (Fig. 6a). Moreover, we knocked down cellular Becn1 using siBecn1 to further show whether the P5 protein affected the expression of BECN1, AMPK, CASP2, AKT, MTOR, and MAPK (ERK, P38). The results showed that there was a significant downregulation of CASP2 and p-AMPK, p-AKT, p-MTOR, and p-MAPK (ERK1/2, P38) levels, and an insignificant alteration of the total amount of these proteins in Becn1-knockdown cells with viral gene $P 5$ transfection (Fig. 6b).

To investigate whether P5 regulated RABV replication depends on the downstream of BECN1-dependent signaling pathway, next we knocked down cellular $A k t$, Mtor, Ampk, Mapk respectively, and examined the NP expression levels in the absence or presence of P5. The results showed that there was a significant decrease of $\mathrm{NP}$ in absence or presence of P5, suggesting that the RABV replication was dependent of the AKT, MTOR, AMPK, MAPK proteins (Fig. 6c). Collectively, these data demonstrated that the BECN1 binding to P5 was responsible for regulating RABV replication via a BECN1mediated signaling pathway.

\section{Discussion}

Previous studies showed that full-length $\mathrm{P}$ protein and at least four additional shorter products P2 (P $\triangle$ N19), P3 (P $\triangle \mathrm{N} 52), \mathrm{P} 4(\mathrm{P} \Delta \mathrm{N} 68)$, and $\mathrm{P} 5$ were detected in RABVinfected cells, viral gene $P$ transfected cells, and purified RABV virions [14]. BECN1 plays an interacting partner 
a
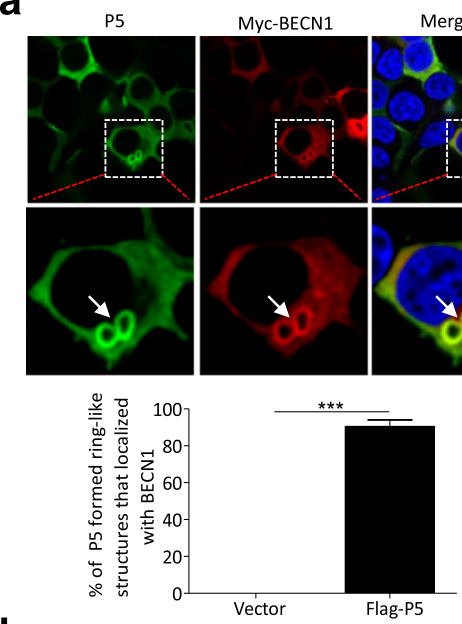

b.

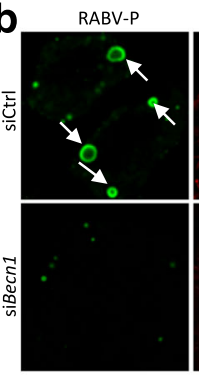

BECN1

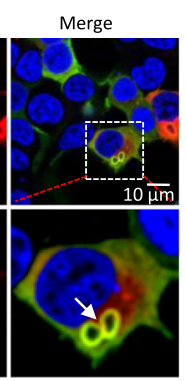

Merge

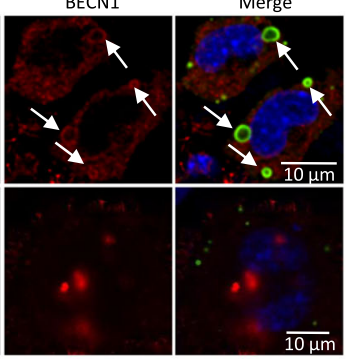

C

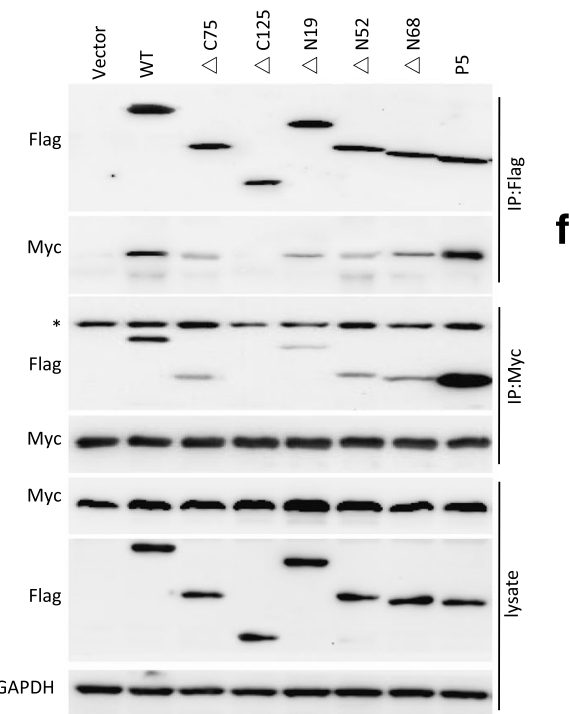

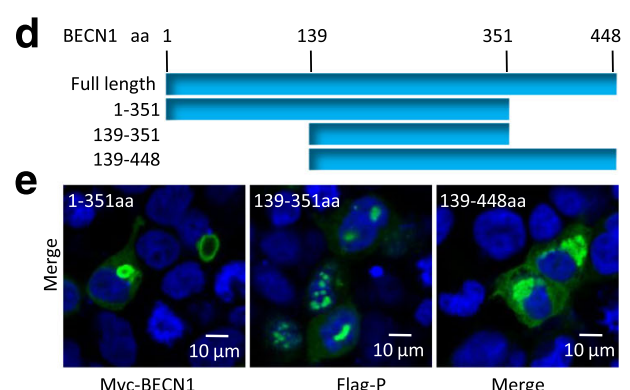
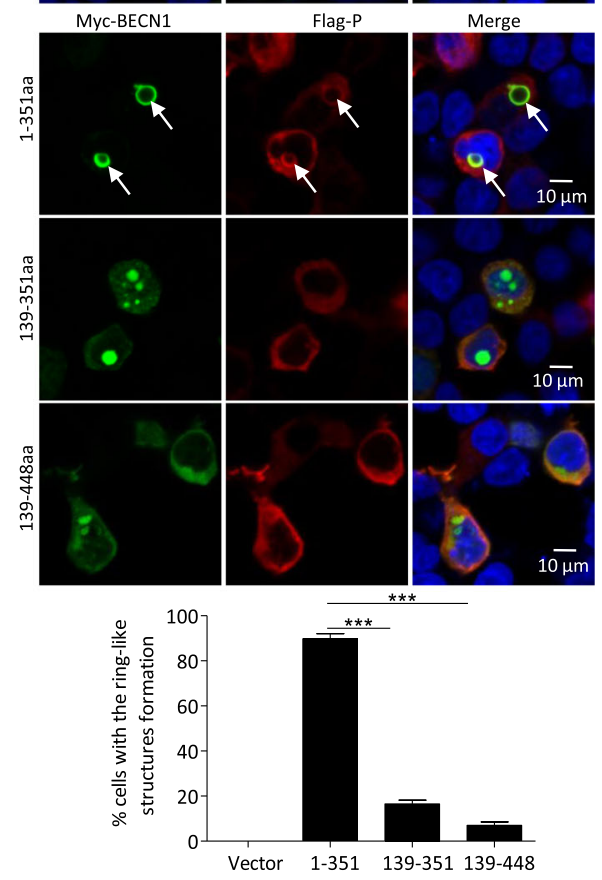

f
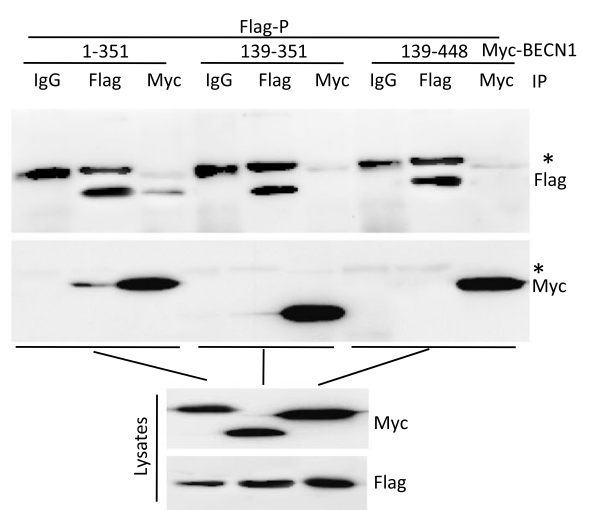

Fig. 4 Truncated protein P5 interacted with BECN1. a, b N2a cells were cotransfected with Flag-P5 and Myc-BECN1 (a) or singly transfected with siBecn 1 RNA for $12 \mathrm{~h}$ and infected with RABV strain HEP-Flury at $\mathrm{MOI}=2$ for $24 \mathrm{~h}(\mathbf{b})$, and the viral P/P5 protein (green), BECN1 (red), and DAPI (blue) were detected using the indicated antibodies via confocal microscopy. White arrows indicate the colocalization of the ring-like structure. Scale bar: $10 \mu \mathrm{m}$. The graph shows the quantification of the percentage of BECN1 localization with P5 ring-like structure. c HEK 293 T cells were cotransfected with the plasmids containing the truncated $P$ genes and Myc-BECN1 for $48 \mathrm{~h}$, and the interactions between the truncated P protein and BECN1 were determined using the indicated antibodies. IP, immunoprecipitation. The asterisk indicates the heavy chains. $\mathbf{d}$ Schematic diagram of the full-length and truncated BECN1 proteins. e N2a cells were singly transfected or cotransfected with the plasmids encoding the truncated BECN1 genes and Flag-P for $24 \mathrm{~h}$, and the viral P protein (red), BECN1 (green), and DAPI (blue) were detected using the indicated antibodies via confocal microscopy. White arrows indicate the colocalization of the ring-like structure. Scale bar: $10 \mu \mathrm{m}$. The graph shows the quantification of the ring-like structures formed with BECN1 deletion mutants. $\mathbf{f H E K} 293 \mathrm{~T}$ cells were cotransfected with the plasmids containing the truncated Becn 1 genes and Flag-P for $48 \mathrm{~h}$, and the interactions between the truncated BECN1 proteins and P were determined using the indicated antibodies. IP, immunoprecipitation. The asterisk indicates the heavy chains. Means and SD (error bars) of three independent experiments are indicated $(* * *, P<0.001)$ 


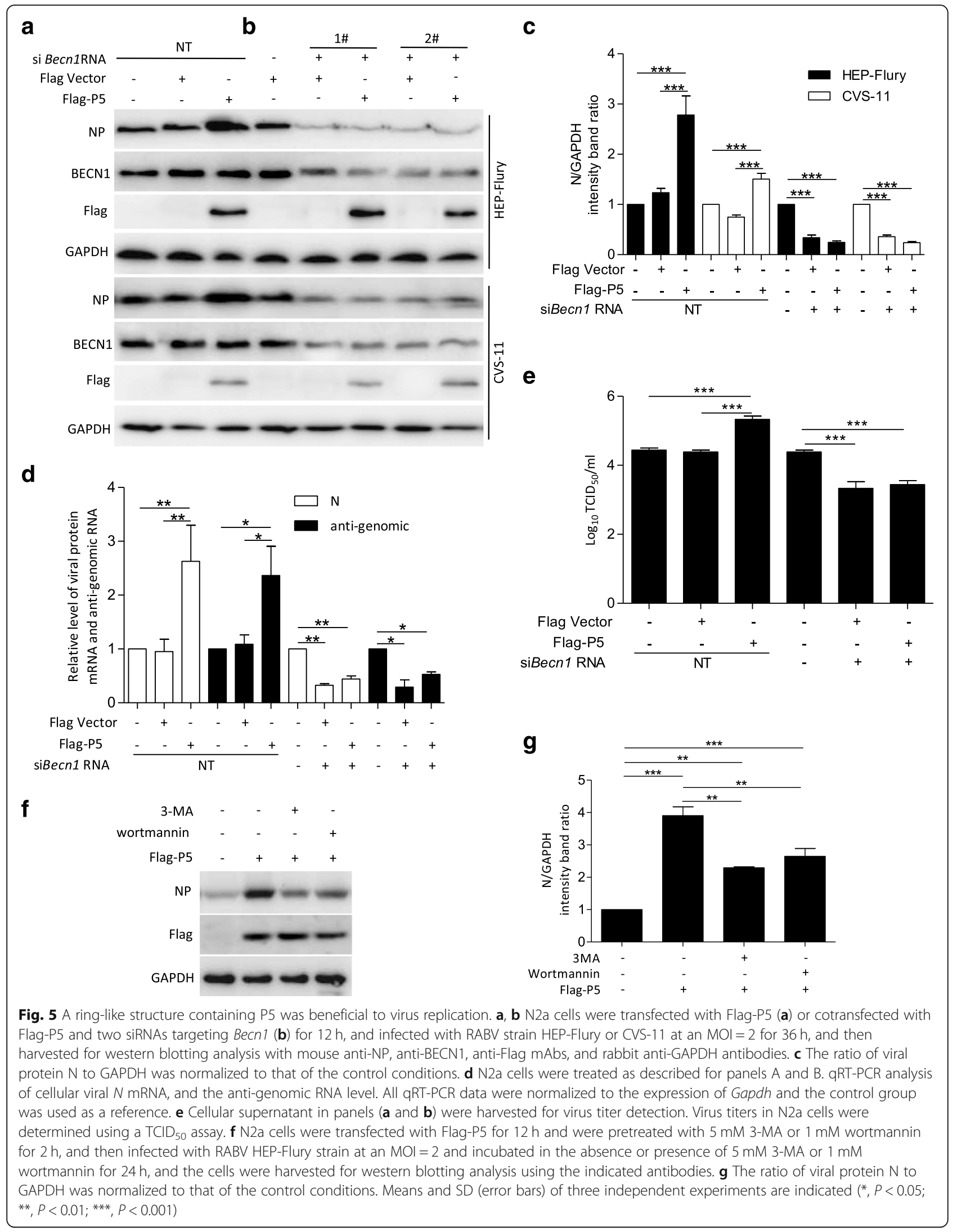




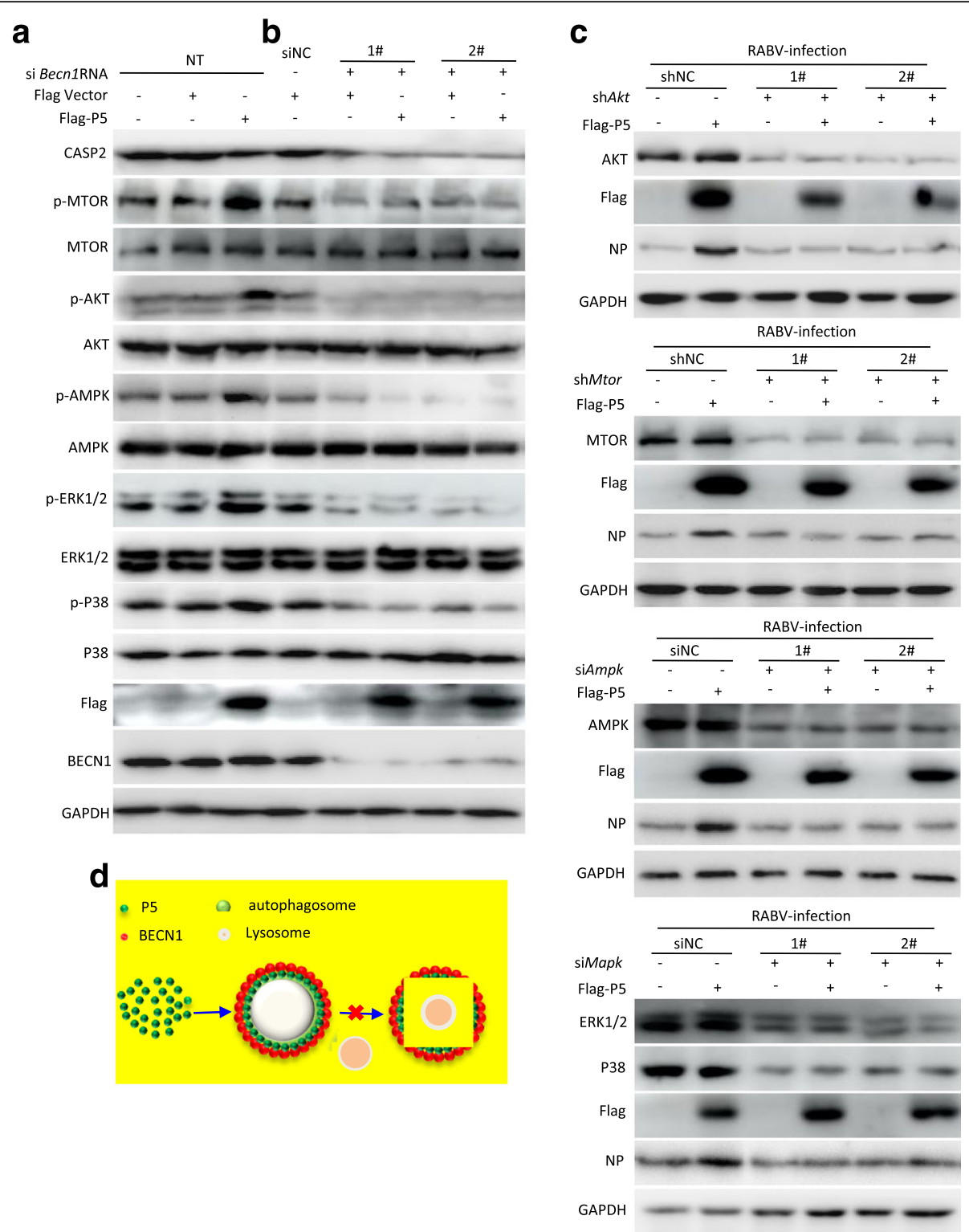

Fig. 6 The protein P5 binding to the BECN1 ring-like structure regulates RABV replication via the BECN1-mediated signaling pathway. a, b N2a cells were transfected with Flag-P5 (a) or cotransfected with Flag-P5 and two siRNAs targeting Becn 1 (b) for $48 \mathrm{~h}$, and then harvested for western blotting analysis with the indicated antibodies. c N2a cells were cotransfected with Flag-P5 and the shRNA/siRNA targeting Akt or Mtor or Ampk or Mapk for $12 \mathrm{~h}$, and infected with RABV HEP-Flury strain at an $\mathrm{MOI}=2$ for $36 \mathrm{~h}$. Cells were harvested for western blotting analysis with the indicated antibodies. $\mathbf{d}$ Proposed model showing how P5 protein regulates RABV replication. Data are representative of 3

independent experiments

role for the mammalian phosphatidylinositol 3-kinase catalytic subunit type 3 (PIK3C3) involving macroautophagy, in which it is an essential chaperone or adaptor [18-20]. However, in the relationship between the virus and autophagy, although it has been reported that BECN1 interacts with a virus protein to regulate autophagy, the specific domain responsible for the BECN1 interaction is not clear $[21,22]$. The present study showed that BECN1 exists in a ring-like structure, and identified that among five truncated $\mathrm{P}$ proteins $(\mathrm{P} \Delta \mathrm{C} 75, \mathrm{P} \Delta \mathrm{N} 19, \mathrm{P} \Delta \mathrm{N} 52, \mathrm{P} \Delta \mathrm{N} 68$, and $\mathrm{P} 5)$, residues $173-222$ induced autophagy by interacting with $\mathrm{N}$-terminal residues $1-139$ of BECN1. Meanwhile, only the full-length $\mathrm{P}$ protein and P5 were visibly colocalized with the BECN1 ring-like structure (Fig. 4). Notably, in co-IP experiments, P5 showed stronger binding than full length $\mathrm{P}$ protein. Therefore, we concluded that RABV small phosphoprotein P5 is responsible for binding to the $\mathrm{BECN1}$ ring-like structure. 
As an essential cofactor of RABV RNA polymerase, $\mathrm{P}$ may participate in additional physiological processes [23]. Our previous research reports incomplete autophagy induced by the RABV phosphoprotein [10]. In this study, we demonstrated that the $\mathrm{P}$ proteins with, but not without, amino acid segment 173-222 are involved in increasing the level of endogenous lipidated LC3-II. In particular, the LC3-II was surrounded by the P5 protein (Figs. 1 and 2). However, P5 did not change the levels of autophagy associated proteins ATG5, ATG7, ULK1, BECN1, and P62, markedly. In addition, the P5-induced autophagosome did not colocalize with lysosomes (Figs. 1, 2 and 3 and S2). Nonetheless, we observed that P5 upregulated the phosphorylation of AMPK, MAPK (P38, ERK1/2), AKT, and MTOR, and decreased BECN1-dependent CASP2 levels (Fig. 6). Collectively, our data demonstrated that amino acid residues 173-222 of the viral $P$ protein are responsible for inducing incomplete autophagy, and the binding of P5 to the $\mathrm{BECN1}$ ring-like structure induced this incomplete autophagy by activating the BECN1 signaling pathway.

Autophagy can remove intracellular pathogens, including bacteria and viruses, by activating various cellular defense responses, including direct digestion of intracytoplasmic virions [21, 24], recognition of pathogenassociated molecular patterns through the delivery of viral genomes to endosomal toll-like receptors [25], activation of innate immune signaling [26], and regulation of the inflammatory response [27-31]. However, many viruses also subvert the autophagic machinery to enhance viral replication [32-37]. In this study, we demonstrated that P5 overexpression increased the level of viral $\mathrm{N}$ protein, viral $N$ mRNA, viral anti-genomic RNA, and infectious RABV progeny, and these indexes were significantly inhibited in the absence or presence of P5 and knockdown of Becn1 (Fig. 5). In addition, we also demonstrated that the P5 still increased the RABV replication when autophagy was inhibited (Fig. 5). These results suggested that RABV replication was regulated by the binding of P5 to the BECN1 ring-like structure. In our previous study, RABV P binding to BECN1 can induce incomplete autophagy through the pathways BECN1-CASP2-AMPK-MAPK and BECN1-CASP2-AMPK-AKT-MTOR and RABV-induced incomplete autophagy provides the scaffolds for the replication of RABV genome. However, in this study, we found that small phosphoprotein P5 binding to BECN1 formed a ring-like structure to induce incomplete autophagy through a BECN1 signaling pathway. The ring-like structure wrapped the immature autophagic vesicles and might prevent the fusion of autophagic vesicles and lysosomes from degrading the RABV, and thus benefited self-replication.

\section{Conclusion}

In conclusion, we identified the binding domain between the RABV phosphoprotein and beclin1, and found that
RABV P5 protein interacted with the BECN1 ring-like structure to induce incomplete autophagy through a $\mathrm{BECN} 1$ signaling pathway. P5 attached to the BECN1 ringlike structure promoted RABV self-replication (Fig. 6d). Thus, the results of the present study identified potential antiviral drug targets against RABV.

\section{Supplementary information}

Supplementary information accompanies this paper at https://doi.org/10. 1186/s12964-020-00644-4.

Additional file 1: Table S1. Primer used for the truncated P or BECN1
protein constructs.

Additional file 2: Figure S1. HEK293T cells were cotransfected with GFP-LC3B and the plasmids containing the truncated $P$ genes for $24 \mathrm{~h}$, and further treated with CQ for $4 \mathrm{~h}$. These cells were fixed, and immunostained with mouse anti-Flag antibodies (red), and then visualized using confocal microscopy. DAPI (blue) was used to stain nuclear DNA. Scale bar: $10 \mu \mathrm{m}$. The graph shows the quantification of autophagosomes by taking the average number of dots in 50 cells. Means and SD (error bars) of three independent experiments are indicated ${ }^{*}, P<0.05$. Figure S2. The truncated protein P5 is required for a ring-like structure. N2a cells were cotransfected with Flag and Myc tagged plasmids encoding the truncated $P$ genes for $24 \mathrm{~h}$, fixed, and immunostained with mouse antiFlag antibody (green) and rabbit anti-Myc (red), and then visualized by confocal microscopy. DAPI (blue) stained nuclear DNA. Scale bar: $10 \mu \mathrm{m}$. Figure S3. Autophagosomes fail to fuse with lysosomes in Flag-P5transfected cells. N2a cells were cotransfected with Flag-P5 and GFP-LC3B for $24 \mathrm{~h}$, and were treated with EBSS or CQ for $4 \mathrm{~h}$. Cells were fixed, and immunostained with rabbit anti-LAMP1 mAb (red), and mouse anti-Flag $\mathrm{mAb}$ (blue), and observed using confocal microscopy to analyze fusion of autophagosomes with lysosomes. Scale bar: $10 \mu \mathrm{m}$. The graph shows the quantification of autolysosomes by taking the average number of dots in 50 cells. Means and SD (error bars) of three independent experiments are indicated $\left({ }^{*}, P<0.05 ;{ }^{*}, P<0.01{ }^{*}{ }^{* *}, P<0.001\right)$. Figure S4. The truncated $P$ proteins colocalize with BECN1. N2a cells were cotransfected with the plasmids encoding the truncated $P$ genes and Myc-BECN1 for $24 \mathrm{~h}$, and Flag (green), BECN1 (red) and DAPI (blue) were detected by using the indicated antibodies in confocal microscopy. Scale bar: $10 \mu \mathrm{m}$.

\section{Abbreviations}

AKT: Protein kinase B; AMPK: AMP-activated protein kinase; ANOVA: Analysis of variance; ATG: Autophagy related; BECN1: Beclin1; CASP2: Caspase2; CoIP: Co-immunoprecipitation; DAPI: 6-diamidino-2-phenylindole; ERK: Extracellular signal-regulated kinase; GAPDH: Glyceraldehyde-3phosphate dehydrogenase; GFP: Green fluorescent protein; LAMP1: Lysosomal associated membrane protein 1; LC3: Microtubule associated protein 1 light chain 3 alpha; mAbs: Mouse monoclonal antibodies; MAPKs: Mitogen activated protein kinases; MOl: Multiplicity of infection; MTOR: Mammalian target of rapamycin; P: Phosphoprotein; RABV: Rabies virus; siRNA: Small interfering RNA

\section{Acknowledgments}

We gratefully acknowledge Ms. Yunqin Li for technical assistance on laser confocal microscopy.

\section{Authors' contributions}

$J Z, J L$ and $M L$ designed the research. $J L$ performed the experiments, analyzed and interpreted data. JL, ML and JZ wrote the manuscript. ML, YY, $\mathrm{HW}$ and HY provide the experimental materials. All authors read and approved the final manuscript.

Funding

This work was supported by grants from National Key Technology R\&D Program of China (2016YFD0500400), and the National Special Fund for Public Welfare Industry (Project No. 201103032) of China. 


\section{Availability of data and materials}

The datasets used and/or analysed during the current study are available from the corresponding author on reasonable request.

\section{Ethics approval and consent to participate}

Not applicable.

\section{Consent for publication}

All authors have read this manuscript and approved for the submission.

\section{Competing interests}

The authors declare no competing interests.

\section{Author details}

${ }^{1}$ MOA Key Laboratory of Animal Virology, Center for Veterinary Sciences, Zhejiang University, 866 Yuhangtang Road, Hangzhou, Zhejiang 310058, PR China. ${ }^{2}$ Collaborative innovation center and State Key laboratory for Diagnosis and Treatment of Infectious Diseases, The First Affiliated Hospital, Zhejiang University, Hangzhou 310058, PR China.

Received: 8 April 2020 Accepted: 10 August 2020 Published online: 18 September 2020

\section{References}

1. Hampson K, Coudeville L, Lembo T, Sambo M, Kieffer A, Attlan M, et al. Estimating the global burden of endemic canine rabies. PLoS Negl Trop Dis. 2015;9:e0003709.

2. Banerjee AK, Barik S, De BP. Gene expression of nonsegmented negative strand RNA viruses. Pharmacol Ther. 1991;51:47-70.

3. Zan J, Liu J, Zhou JW, Wang HL, Mo KK, Yan Y, et al. Rabies virus matrix protein induces apoptosis by targeting mitochondria. Exp Cell Res. 2016; 347:83-94.

4. Chelbi-Alix MK, Vidy A, El Bougrini J, Blondel D. Rabies viral mechanisms to escape the IFN system: the viral protein P interferes with IRF-3, Stat1, and PML nuclear bodies. J Interf Cytokine Res. 2006;26:271-80.

5. Jacob Y, Badrane H, Ceccaldi PE, Tordo N. Cytoplasmic dynein LC8 interacts with lyssavirus phosphoprotein. J Virol. 2000;74:10217-22.

6. Kammouni W, Wood H, Saleh A, Appolinario CM, Fernyhough P, Jackson AC. Rabies virus phosphoprotein interacts with mitochondrial complex I and induces mitochondrial dysfunction and oxidative stress. J Neuro-Oncol. 2015;21:370-82

7. Fouquet B, Nikolic J, Larrous F, Bourhy H, Wirblich C, Lagaudriere-Gesbert C, et al. Focal adhesion kinase is involved in rabies virus infection through its interaction with viral phosphoprotein P. J Virol. 2015;89:1640-51.

8. Blondel D, Regad T, Poisson N, Pavie B, Harper F, Pandolfi PP, et al. Rabies virus $P$ and small $P$ products interact directly with $P M L$ and reorganize $P M L$ nuclear bodies. Oncogene. 2002;21:7957-70.

9. Xu Y, Liu F, Liu J, Wang D, Yan Y, Ji S, et al. The co-chaperone Cdc37 regulates the rabies virus phosphoprotein stability by targeting to Hsp90AA1 machinery. Sci Rep. 2016;6:27123.

10. Liu J, Wang H, Gu J, Deng T, Yuan Z, Hu B, et al. BECN1-dependent CASP2 incomplete autophagy induction by binding to rabies virus phosphoprotein. Autophagy. 2017;13:739-53.

11. Vidy A, Chelbi-Alix M, Blondel D. Rabies virus P protein interacts with STAT and inhibits interferon signal transduction pathways. J Virol. 2005;79:14411-20.

12. Lieu KG, Brice A, Wiltzer $L$, Hirst $B$, Jans DA, Blondel D, et al. The rabies virus interferon antagonist $P$ protein interacts with activated STAT3 and inhibits Gp130 receptor signaling. J Virol. 2013;87:8261-5.

13. Gupta AK, Blondel D, Choudhary S, Banerjee AK. The phosphoprotein of rabies virus is phosphorylated by a unique cellular protein kinase and specific isomers of protein kinase C. J Virol. 2000;74:91-8.

14. Chenik M, Chebli K, Blondel D. Translation initiation at alternate in-frame AUG codons in the rabies virus phosphoprotein mRNA is mediated by a ribosomal leaky scanning mechanism. J Virol. 1995;69:707-12.

15. Pasdeloup D, Poisson N, Raux H, Gaudin Y, Ruigrok RW, Blondel D. Nucleocytoplasmic shuttling of the rabies virus $P$ protein requires a nuclear localization signal and a CRM1-dependent nuclear export signal. Virology. 2005;334:284-93.

16. Zhang J, Ruan X, Zan J, Zheng X, Yan Y, Liao M, et al. Efficient generation of monoclonal antibodies against major structural proteins of rabies virus with suckling mouse brain antigen. Monoclonal Antibodies Immunodiagnosis Immunother. 2014:33:94-100.

17. Zhang J, Wu X, Zan J, Wu Y, Ye C, Ruan X, et al. Cellular chaperonin CCTgamma contributes to rabies virus replication during infection. J Virol. 2013;87:7608-21.

18. Petiot A, Ogier-Denis E, Blommaart EF, Meijer AJ, Codogno P. Distinct classes of phosphatidylinositol 3'-kinases are involved in signaling pathways that control macroautophagy in HT-29 cells. J Biol Chem. 2000;275:992-8.

19. Zeng X, Overmeyer JH, Maltese WA. Functional specificity of the mammalian Beclin-Vps34 PI 3-kinase complex in macroautophagy versus endocytosis and lysosomal enzyme trafficking. J Cell Sci. 2006;119:259-70.

20. Strappazzon F, Vietri-Rudan M, Campello S, Nazio F, Florenzano F, Fimia GM, et al. Mitochondrial BCL-2 inhibits AMBRA1-induced autophagy. EMBO J. 2011;30:1195-208.

21. Orvedahl A, Alexander D, Talloczy Z, Sun Q, Wei Y, Zhang W, et al. HSV-1 ICP34.5 confers neurovirulence by targeting the Beclin 1 autophagy protein. Cell Host Microbe. 2007;1:23-35.

22. Gannage M, Dormann D, Albrecht R, Dengjel J, Torossi T, Ramer PC, et al. Matrix protein 2 of influenza a virus blocks autophagosome fusion with lysosomes. Cell Host Microbe. 2009;6:367-80.

23. Marschalek A, Drechsel L, Conzelmann KK. The importance of being short: the role of rabies virus phosphoprotein isoforms assessed by differential IRES translation initiation. Eur J Cell Biol. 2012;91:17-23.

24. Talloczy Z, Virgin HW, Levine B. PKR-dependent autophagic degradation of herpes simplex virus type 1. Autophagy. 2006;2:24-9.

25. Delgado MA, Elmaoued RA, Davis AS, Kyei G, Deretic V. Toll-like receptors control autophagy. EMBO J. 2008;27:1110-21.

26. Shi CS, Kehrl JH. MyD88 and Trif target Beclin 1 to trigger autophagy in macrophages. J Biol Chem. 2008;283:33175-82.

27. Lupfer C, Thomas PG, Anand PK, Vogel P, Milasta S, Martinez J, et al. Receptor interacting protein kinase 2-mediated mitophagy regulates inflammasome activation during virus infection. Nat Immunol. 2013;14:480-8.

28. Zhang M, Covar J, Zhang NY, Chen W, Marshall B, Mo J, et al. Virus spread and immune response following anterior chamber inoculation of HSV-1 lacking the Beclin-binding domain (BBD). J Neuroimmunol. 2013;260:82-91.

29. Saitoh T, Fujita N, Jang MH, Uematsu S, Yang BG, Satoh T, et al. Loss of the autophagy protein Atg16L1 enhances endotoxin-induced IL-1 beta production. Nature. 2008;456:264-8.

30. Paludan C, Schmid D, Landthaler M, Vockerodt M, Kube D, Tuschl T, et al. Endogenous MHC class II processing of a viral nuclear antigen after autophagy. Science (New York, NY). 2005;307:593-6.

31. English L, Chemali M, Duron J, Rondeau C, Laplante A, Gingras D, et al. Autophagy enhances the presentation of endogenous viral antigens on MHC class I molecules during HSV-1 infection. Nat Immunol. 2009;10:480-7.

32. Wong J, Zhang J, Si X, Gao G, Mao I, McManus BM, et al. Autophagosome supports coxsackievirus B3 replication in host cells. J Virol. 2008:82:9143-53.

33. Lee YR, Lei HY, Liu MT, Wang JR, Chen SH, Jiang-Shieh YF, et al. Autophagic machinery activated by dengue virus enhances virus replication. Virology. 2008;374:240-8

34. Panyasrivanit M, Khakpoor A, Wikan N, Smith DR. Co-localization of constituents of the dengue virus translation and replication machinery with amphisomes. J Gen Virol. 2009;90:448-56.

35. Kyei GB, Dinkins C, Davis AS, Roberts E, Singh SB, Dong C, et al. Autophagy pathway intersects with HIV-1 biosynthesis and regulates viral yields in macrophages. J Cell Biol. 2009;186:255-68.

36. Denizot M, Varbanov M, Espert L, Robert-Hebmann V, Sagnier S, Garcia E, et al. HIV-1 gp41 fusogenic function triggers autophagy in uninfected cells. Autophagy. 2008;4:998-1008.

37. Zhou Z, Jiang X, Liu D, Fan Z, Hu X, Yan J, et al. Autophagy is involved in influenza a virus replication. Autophagy. 2009;5:321-8.

\section{Publisher's Note}

Springer Nature remains neutral with regard to jurisdictional claims in published maps and institutional affiliations. 